\title{
Myelodysplastic syndrome patient-derived xenografts: from no options to many
}

Haematologica 2020

Volume 105(4):864-869

\section{Correspondence:}

BO T. PORSE

bo.porse@finsenlab.dk

Received: October 9, 2019.

Accepted: November 27, 2019.

Pre-published: March 19, 2020.

doi:10.3324/haematol.2019.233320

Check the online version for the most updated information on this article, online supplements, and information on authorship \& disclosures: www.haematologica.org/content/105/4/864

\section{(C)2020 Ferrata Storti Foundation}

Material published in Haematologica is covered by copyright. All rights are reserved to the Ferrata Storti Foundation. Use of published material is allowed under the following terms and conditions:

https://creativecommons.org/licenses/by-nc/4.0/legalcode. Copies of published material are allowed for personal or internal use. Sharing published material for non-commercial purposes is subject to the following conditions: https://creativecommons.org/licenses/by-nc/4.0/legalcode, sect. 3. Reproducing and sharing published material for commercial purposes is not allowed without permission in writing from the publisher.

\author{
Christophe Côme,,$^{1,2,3}$ Alexander Balhuizen, ${ }^{1,2,3}$ Dominique Bonnet $^{4}$ \\ and Bo T. Porse ${ }^{1,2,3}$
}

${ }^{1}$ The Finsen Laboratory, Rigshospitalet, Faculty of Health Sciences, University of Copenhagen, Denmark; 'Biotech Research and Innovation Center (BRIC), University of Copenhagen, Copenhagen, Denmark; ${ }^{3}$ Danish Stem Cell Center (DanStem), Faculty of Health Sciences, University of Copenhagen, Denmark and ${ }^{4}$ Haematopoietic Stem Cell Laboratory, The Francis Crick Institute, London, UK

\section{Introduction}

According to the recently updated tumor classification by the World Health Organization, myelodysplastic syndrome (MDS) constitutes a heterogeneous group of blood disorders characterized by cytopenia and dysplasia in at least one of the myeloid lineages. ${ }^{1} \mathrm{MDS}$ is most common in the elderly and is caused by inefficient hematopoiesis and increased apoptosis within the bone marrow (BM). It is a genetically heterogeneous disorder and individual cases generally harbor two to three mutations in one of approximately 30 driver genes which are recurrently mutated in MDS. ${ }^{2,3}$ Of importance, many of these genes have also been found to be mutated in acute myeloid leukemia (AML), with frequencies of mutations differing between the two diseases. ${ }^{2,4}$ The spectrum of survival of patients with MDS is broad and high-risk MDS is associated with an increased propensity to progression to AML. ${ }^{5}$

There has been considerable emphasis on the development of genetically engineered mouse models in attempts to study MDS. These include strains harboring lesions in the most commonly mutated genes in MDS, such as SF3B1, ${ }^{6}$ TET2,,8 ASXL19 and SRSF2. ${ }^{10}$ The phenotypic properties of these models have been reviewed in detail previously ${ }^{11-13}$ and although they all present with several phenotypic features of MDS, they clearly have some limitations with respect to their abilities to recapitulate human MDS biology. As an example, $S f 361^{K 700 E}$ mutant mice develop anemia and display expansion of the long-term hematopoietic stem cell compartment, consistent with an MDS phenotype. However, the $S f 361^{K \gamma 00 E}$ mutant line fails to present with ring sideroblasts which are normally found in patients with SF3B1 mutations. ${ }^{14}$ Another likely contributor to the inability of current genetically engineered mouse model lines to fully recapitulate the phenotypic spectrum of MDS is the fact that most models typically harbor one genetic lesion and, therefore, not the full mutational complement observed in MDS patients. Thus, there is a clear need for better models of MDS biology, including patient-derived xenografts (PDX), in order to recapitulate the disease's biology and complexity better.

\section{The history of myelodysplastic syndrome patient-derived xenografts}

The first PDX models of AML were established more than 40 years ago by subcutaneously engrafting patient material into immune-deprived mice. ${ }^{15}$ More physiologically relevant models were developed over the next decade via the use of tail vein injection and improved immune-deficient strains. ${ }^{16,17}$ In contrast, it was not until the beginning of this millennium that cells from MDS patients were demonstrated to engraft functionally in immune-compromised mice. ${ }^{18-20}$ However, only cells from a limited number of patients could be engrafted and a study with a large number of patients demonstrated that engraftment was sustained by residual normal cells and not by the MDS clone(s). ${ }^{21}$ During the last decade, several laboratories have published a number of complementary approaches for the generation of MDS PDX. ${ }^{22-34}$ Importantly, these combined efforts have demonstrated the engraftment capacity of most MDS subtypes, , $3-28,34^{2}$ that the expanded cells retain the genetic and phenotypic features of the primary tumor, ${ }^{2427,29,30,32,34}$ that these PDX models also sustain engraftment in secondary recipients $s^{24,27,29,34}$ and that they allow evaluation of new therapies. ${ }^{32,33}$ Nevertheless, as summarized in Table 1 and Figure 1, these models are quite heterogeneous. Specifically, several immune-compromised murine strains have been used (NOG, NSG, NSG-S or MISTRG) and injected at different ages (from newborn pups to adult animals). Moreover, a number of different cell 
Table 1. Summary of published patient-derived xenograft models from myelodysplastic syndrome patients.

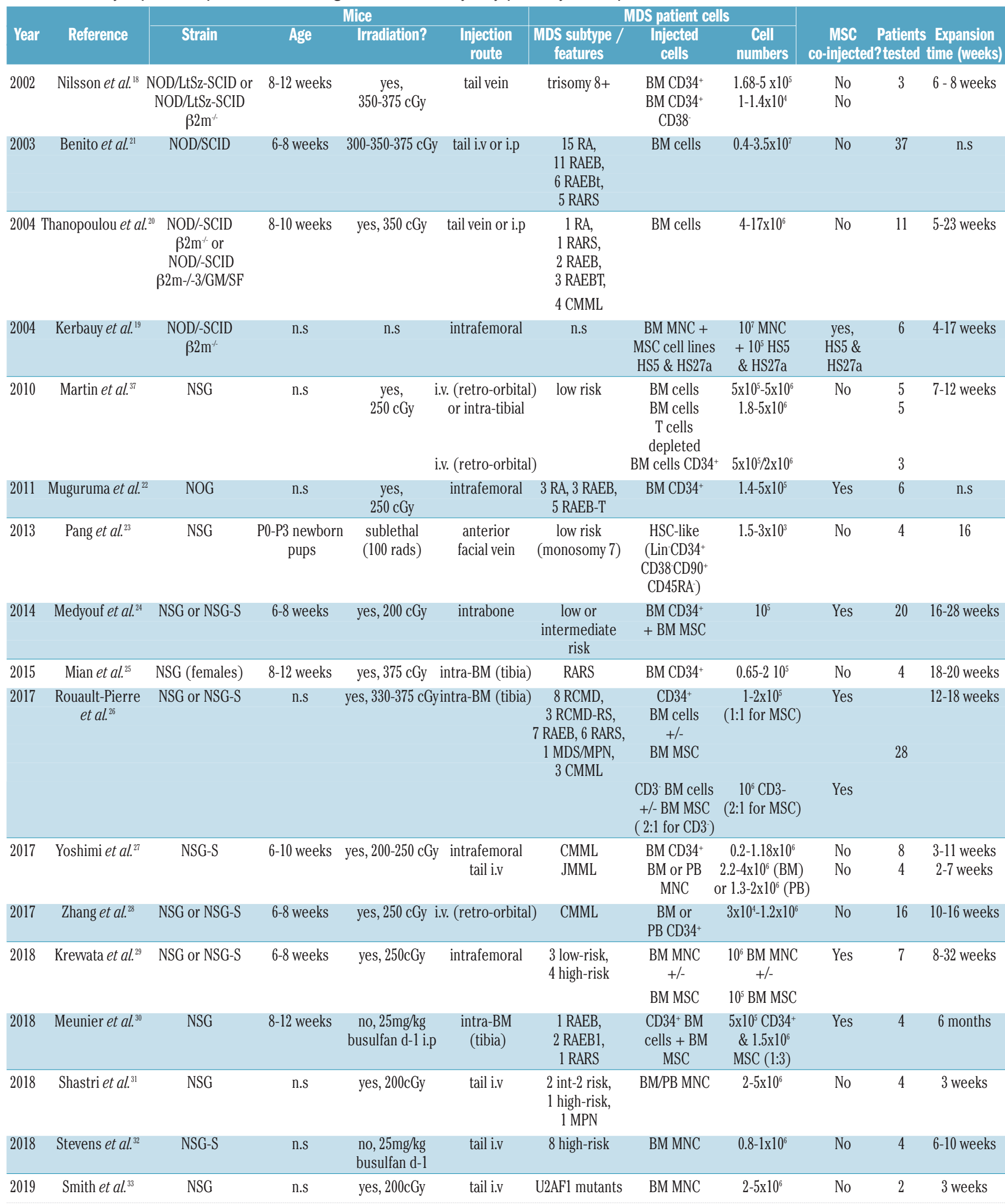


1 del $(5 q)$,

3 MLD,

2 RS-SLD,

1 RS-MLD,

1 MPN-RS-T,

3 EB-1, 3 EB-2

1 MLD, 2 EB-1,

\section{$1 \mathrm{~EB}-2$}

\author{
$\begin{array}{lllll}\text { BM CD }^{+} 4^{+} & 0.2-2.75 \times 10^{5} & \text { No } & 22 & 12-31 \text { weeks }\end{array}$ \\ BM CD3- $\quad 1.15-6 \times 10^{5} \quad$ No $\quad 4 \quad 13-15$ weeks
}

The main characteristics of the protocols published to generate patient-derived xenograft (PDX) models from patients with myelodysplastic syndromes (MDS) are presented. NOD/SCID: nonobese diabetic mice with Prkdc sid mutation; $\beta 2 \mathrm{~m}^{\prime}$ : beta2-microglobulin null; $3 / \mathrm{GM} / \mathrm{SF}$ : constitutive expression of human interleukin-3 (IL-3), granulocyte-macrophage colony-stimulating factor

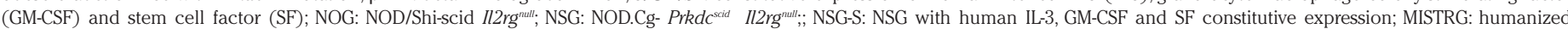
macrophage colony-stimulating factor, IL-3/GM-CSFSIRP alpha and thrombopoietin combined with Rag2 2 IL2R $\gamma$; i.v: intravenous injection; i.p: intraperitoneal injection; cGy: centigray; BM: bone marrow; PB: peripheral blood; MNC: mononuclear cells; MSC: mesenchymal stromal cells; HSC: hematopoietic stem cells; Lin-: lineage negative; RA: refractory anemia; RAEB: refractory anemia with excess blasts; RAEB-I: RAEB type 1; RAEB-II: RAEB type 2; RAEBt: RAEB in transformation; RARS: refractory anemia with ringed sideroblasts; RARS-T: RARS with thrombocytosis; RS: ringed sideroblasts; RCMD: refractory cytopenia with multilineage dysplasia; RCMD-RS: RCMD and ringed sideroblasts; del(5q): MDS associated with isolated deletion of chromosome 5q; MDS/MPN: myelodysplastic/myeloproliferative neoplasms; CMML: chronic myelomonocytic leukemia; JMML: juvenile myelomonocytic leukemia; MLD: multilineage dysplasia; SLD: single lineage dysplasia; EB: excess blasts; MDS/MPN-RS-T: MDS/MPN with ring sideroblasts and thrombocytosis; n.s.: not specified.

sources have been employed (BM or peripheral blood mononuclear cells, CD3-depleted BM cells, CD34+ BM cells) which were injected in different quantities, in the presence or absence of BM-derived mesenchymal stromal cells (MSC) and in different anatomical locations (intravenous, intrafemoral, intrahepatic). Not surprisingly, this resulted in very different disease latencies (from 3 to 32 weeks post-injection). A number of conclusions can be drawn from this extensive work:

(i) With respect to selection of the recipient strain, an immunodeficient background is necessary. The most commonly used recipient for the generation of PDX is the NSG strain which harbors mutations in Prkdc and $I l 2 g$ leading to the absence of $\mathrm{B}, \mathrm{T}$ and NK cells. ${ }^{35}$ The constitutive expression of the human cytokines interleukin-3 (Il3), granulocyte-macrophage colony-stimulating factor (GM-CSF) and stem cell factor (SCF) on this background (NSG-S, also designated NSG-SGM3) does not lead to enhanced engraftment of most MDS subtypes, except for chronic myelomonocytic leukemia, ${ }^{28}$ in contrast to the situation in AML. ${ }^{24,26,29,36}$ On the other hand, the recently developed MISTRG strain, expressing human macrophage colony-stimulating factor (M-CSF), IL-3, GM-CSF, signal regulatory protein alpha (SIRP $\alpha$ ) and thrombopoietin at physiological levels on a different immunodeficient background (Rag $2^{-}$, IL $2 \mathrm{R} \gamma^{-/}$), was recently demonstrated to be a promising host for engraftment of MDS patients' material. ${ }^{34}$ Not only could cells from patients with various subtypes of MDS be expanded in this line, but the levels of engraftment were increased, with a higher percentage of

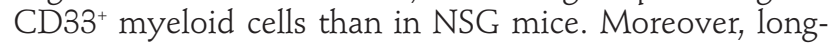
term engraftment of these myeloid cells was also improved in this strain as $\mathrm{CD}^{+} 3^{+}$cells constituted more than $80 \%$ of the hCD $45^{+}$compartment in secondary recipients, compared to $30 \%$ in NSG mice. Additionally, MDS cells engrafted in MISTRG mice generated erythroid and megakaryocytic lineages at a higher frequency than in the NSG counterpart. ${ }^{34}$

(ii) T-cell depletion of the primary MDS tumor, either by treatment with a human CD3 antibody or by physical separation, is a prerequisite to limit graft-versus-host disease. ${ }^{26,34,36-38}$ Indeed, one of the first attempts to generate MDS PDX failed mainly because of the predominant growth of human $\mathrm{CD}^{+} \mathrm{T}$ cells, leading to graft-versus-host disease in most of the recipient animals. ${ }^{37}$

(iii) Intrafemoral injections result in better engraftment in NSG mice compared to an intravenous route of injection. (iv) Co-injection of MSC leads to variable results in terms of promoting the engraftment of MDS samples, with some laboratories reporting some enhancement, ${ }^{22-24}$ whereas others have not found this effect. ${ }^{26,29}$ The underlying reasons for this variation are not clear. However, as human MSC only survive for 2-4 weeks in the murine $\mathrm{BM},{ }^{24,26}$ this variation could potentially reflect patientspecific differences in the ability of MSC to promote the initial seeding and engraftment of MDS cells in the murine BM.

(v) Engraftment capacity does not seem to be related to MDS subtypes, but rather appears to be specific to the individual samples, as indicated in studies with large numbers of patients. . $^{4,26,34}$

\section{Alternative strategies}

Despite extensive efforts in several laboratories, this cumulative work has only produced a total of approximately 100 MDS PDX so far. There is, therefore, a strong need for alternative systems that could enhance the generation of MDS PDX. Interestingly, descriptions of a number of humanized bone marrow-like structure (hBMLS) models have been published recently. These models enable the expansion of AML patients' cells that failed to engraft with conventional methods. ${ }^{39-41}$ They are all based on the use of BM MSC and can be separated into two categories. In the first category, which we will define as "scaffold" models, in vitro-expanded MSC are seeded in a gelatin sponge and cultured for a couple of days. Next, human leukemic cells are injected into the sponge which is subsequently introduced subcutaneously into non-irradiated immunocompromised mice ${ }^{41}$ (Figure 1). In the second approach, BM MSC are first mixed with Matrigel and introduced subcutaneously into immune-deficient mice in which they develop a so-called "ossicle" after 2-3 months, which constitutes an exterior bone structure surrounding a hematopoietic core. Following sublethal irradiation, human leukemic cells are injected into the ossicle where they expand ${ }^{39}$ (Figure 1). Another ossicle-like approach combines osteogenic priming of MSC with a physical support consisting of two or three biphasic calcium phosphate particles, prior to subcutaneous insertion into mice and subsequent ossicle development..$^{40}$ Importantly, up to four hBMLS per animal can be introduced, ${ }^{39-41}$ and Reinisch et al. have elegantly demonstrated that tumor cells can cir- 


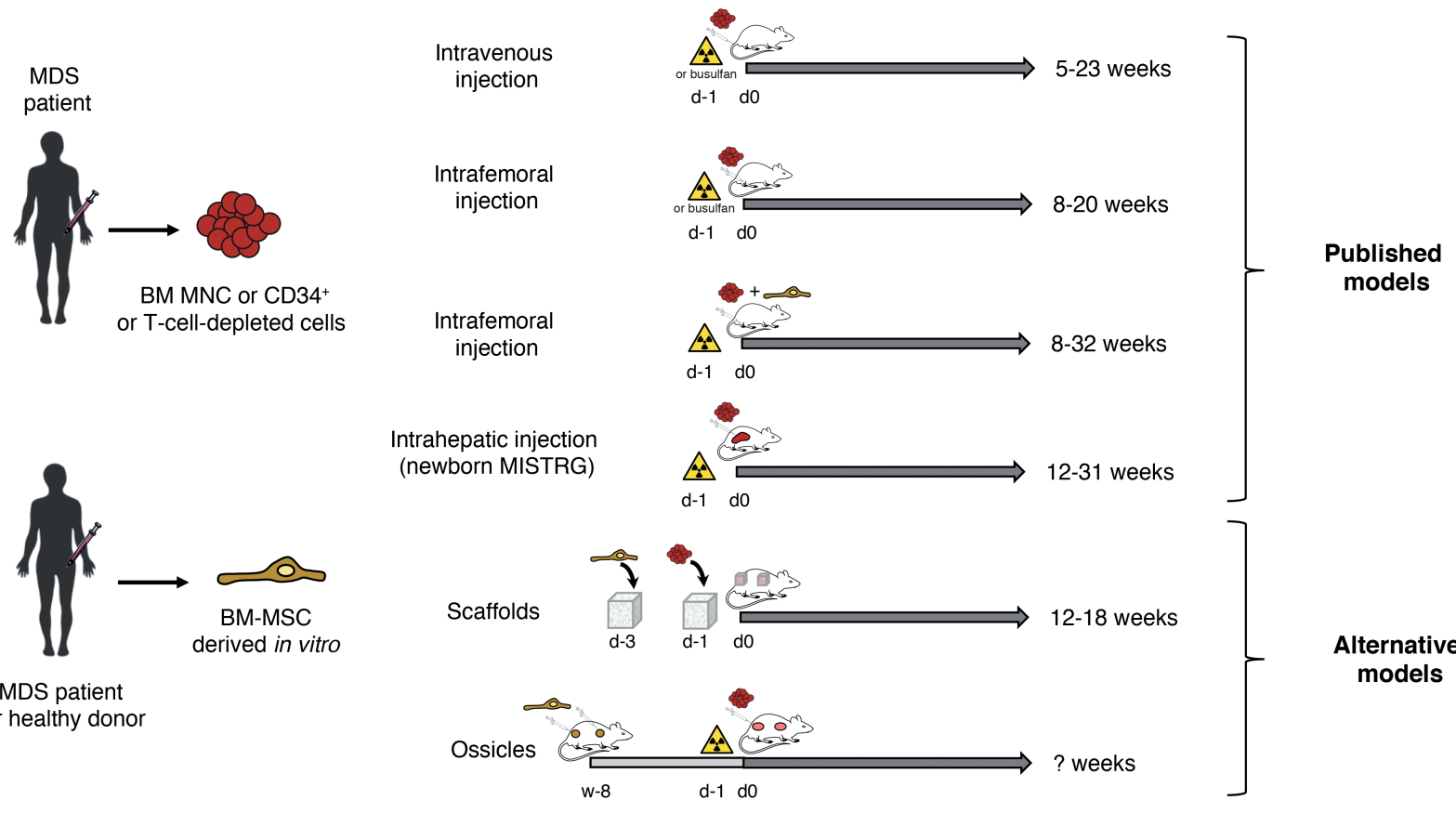

Mouse sublethal irradiation

Gelatin sponge

ㅁ In vivo ossicle generation by MSC

$\Rightarrow$ In vivo expansion of MDS cells

Figure 1. Key features of published and alternative patient-derived xenograft models of myelodysplastic syndrome. The left panel depicts the sources of cells from patients with myelodysplastic syndrome (MDS) which are injected to generate MDS patient-derived xenografts. Tumor cells (red circles) are constituted of bone marrow (BM) cells, mononuclear cells (MNC), or CD34+ purified or T-cell depleted BM cells. Supporting cells (yellow) are BM-derived mesenchymal stromal cells (MSC) derived from patients or healthy donors. The time periods for ossicle development and engraftment of MDS cells are illustrated by light and dark gray bars, respectively. The time of conditioning of the animal, by either irradiation or busulfan treatment is indicated, and the injection route is illustrated by a syringe.

culate between ossicles leading to engraftment of leukemic cells in non-injected hBMLS, thereby allowing for increased expansion of the original material from patients. ${ }^{39}$

In the previously described MDS PDX models, engraftment and expansion of the MDS material occur mainly in the recipient $B M$. In contrast, the hBMLS approaches exploit a humanized version of the $\mathrm{BM}$ niche, since at least bone, cartilage and MSC present in the niche are of human origin. ${ }^{42}$ Of note, these hBMLS constitute a preferential homing niche for leukemic cells when compared to murine BM because leukemic cells injected intravenously expand earlier and at higher frequency in hBMLS than in the BM of mice. ${ }^{39,40}$ Moreover, as the BM microenvironment has been reported to play an important role in the onset and development of MDS as well as the response to therapy, these hBMLS models are likely to be superior in mimicking key disease parameters. ${ }^{43,44}$

\section{Is a standardized approach possible?}

As discussed above, a plethora of approaches has been or could be used to generate PDX from MDS patients (Table 1 and Figure 1). However, these approaches are quite heterogeneous, and use different murine strains, injection sites, types and numbers of cells injected. In order to facilitate a comparison between different studies, it would be helpful if the field could agree on a more limited set of robust experimental protocols. In our opinion, two options are quite attractive. Our first candidate is the MISTRG model which has been demonstrated to mediate the engraftment of material from patients with different subtypes of MDS and appears relatively simple to implement. Moreover, in the published research, in which patients' cells have been injected intrahepatically into irradiated pups, this line appears to be superior to NSG in terms of engraftment frequency and myeloid percentages. ${ }^{34}$ One note of caution is the reported development of anemia in this strain, which is also a characteristic of human MDS. ${ }^{45,46}$ This may potentially make it complicated to determine whether the anemia observed in MDS PDX is caused by defects in MDS hematopoietic stem cells or by the intrinsic phenotype of the MISTRG strain. Moreover, the intrahepatic route of injection in newborn pups may not only raise some logistic challenges, but could also potentially influence tumor behavior, because this system constitutes a "young" niche, in contrast to the BM niche of elderly MDS patients. It is to be hoped that further generation of AML/MDS PDX with this mouse model by additional laboratories will strengthen the relevance of this model.

Even though the ossicle strategy is extremely seducing as it allows engraftment of patients' cells into a mature humanized BM-like environment, our own experience indicates that a very high proportion of MSC batches fail to sustain ossicle development (11/12, unpublished observa- 
tions). Moreover, to our knowledge, AML PDX models based on this approach have only been described by one laboratory so far. ${ }^{39,42}$ Therefore, our second proposed model is hBMLS based on gelatin scaffolds. This technique is quite simple and, as for ossicles, up to four scaffolds can be inserted per animal. Moreover, this strategy does not involve a long period of in vivo incubation in order to generate ossicles and, importantly, does not require pre-conditioning with irradiation. ${ }^{41}$ Using this technique, we have succeeded in generating MDS PDX models covering several MDS subtypes in both our laboratories. A limitation of this approach, as for other hBMLS models, is the use of BM-derived MSC because these MSC have various alterations compared to those derived from healthy donors, such as DNA methylation status ${ }^{47,48}$ and in vitro proliferation/differentiation capacity. ${ }^{47}$ There is therefore a risk that the use of healthy allogeneic MSC may affect the behavior of the MDS clone(s) in vivo. Encouragingly, the few studies that have compared the use of healthy and patient-specific MSC have not suggested a major impact of the MSC origin on the engraftment levels of $\mathrm{MDS}$ in immunocompromised mice receiving intra-femoral injections. ${ }^{26,29}$ Nevertheless, MDS-derived BM MSC do have an impact on the survival and differentiation capacities of $\mathrm{CD}^{3} 4^{+}$hematopoietic stem and progenitor cells in vitro and in vivo, ${ }^{47,49}$ and they can also respond favorably to the hypomethylating agent azacytidine, the current treatment regimen for high-risk MDS. ${ }^{49}$ Consequently, investigation are needed to determine whether autologous MDS-BM MSC would be better at recapitulating the complexity of the disease in this model rather than BM MSC from healthy donors.

A major unresolved issue for the hBMLS approaches is that MSC display significant donor-to-donor variations and it would therefore be extremely useful to have a standardized source of MSC, i.e. in the form of BM MSC lines. Importantly, such cell lines have been generated recently and it would be of paramount importance to determine whether they retain their capacity to generate hBMLS in vivo $0^{50}$ and whether MDS material could engraft and expand in these structures. As MDS MSC have been shown to have a strong impact on the in vivo potential of $\mathrm{CD}_{3} 4^{+}$hematopoietic stem and progenitor cells, notably by showing altered extracellular signaling such as reduced CXCL12 expression, ${ }^{48,49}$ such a cell line should either retain the features of MDS MSC or be receptive to "education" by MDS cells. However, if a MSC cell line that robustly retains these features could be obtained, this would provide an experimental platform for genetic manipulation of niche-derived cells, thereby facilitating studies into niche-MDS cell interactions.

\section{Conclusions and perspectives}

MDS is a very heterogeneous group of blood disorders, associated with lesions in dozens of driver genes. ${ }^{2,3}$ Genetically engineered mouse models harboring mutations in the most common MDS driver genes display several characteristics of $\mathrm{MDS}^{11-13}$ but remain imperfect as an experimental tool since they generally only recapitulate a subset of the phenotypes associated with human MDS. During the past few decades, in particular during the past 5 years, we have seen several improvements in the toolbox available for the generation of MDS PDX. . $^{182,22-27,29,31,34}$ Moreover, various alternative methods, especially hBMLS models, appear to be extremely promising in terms of facilitating a more robust generation of MDS PDX. ${ }^{39-41}$ This is important since an increase in the number of MDS PDX models will allow us to cover the broad genetic and phenotypic spectra of human MDS more comprehensively and provide tools to address key aspects of MDS biology.

Despite the recent developments in MDS PDX, these models may be further improved by incorporating additional human niche cells, such as endothelial cells. Indeed, these cells are functional in hBMLS settings ${ }^{51,52}$ and endothelial cells from low-risk MDS patients influence hematopoietic stem cell behavior in vitro. ${ }^{53}$ However, the recent developments of hBMLS models already provide an excellent opportunity to characterize the interaction between MDS tumor cells and their microenvironment better. As indicated above, the tumor microenvironment plays a key role in the pathogenesis of MDS and if we could manipulate MSC in the hBMLS models, we would have a precise tool to discern the biological importance of the niche. Finally, the increasing armory of MDS PDX also holds great promise as preclinical translational models for the development and validation of novel therapies as well as for personalized medicine along the lines already occurring in solid cancers.

\section{Acknowledgement \\ Work in the Porse laboratory was supported through a center grant from the Novo Nordisk Foundation (Novo Nordisk Foundation Center for Stem Cell Biology, DanStem; Grant Number NNF17CCO027852). The present work is also part of the Danish Research Center for Precision Medicine in Blood Cancers funded by the Danish Cancer Society (grant n. R223- A13071) and Greater Copenhagen Health Science Partners. Work in the Bonnet laboratory was supported by the Francis Crick Institute, which receives its core funding from Cancer Research UK (FC001045), The UK Medical Research Council (FC001045) and the Wellcome Trust (FC001045).}

\section{References}

1. Arber DA, Orazi A, Hasserjian R. The 2016 revision to the World Health Organization classification of myeloid neoplasms and acute leukemia . 2016;127(20):2391-2405

2. Papaemmanuil E, Gerstung M, Malcovati L, et al. Clinical and biological implications of driver mutations in myelodysplastic syndromes. Blood. 2013;122(22):3616-3627.

3. Haferlach T, Nagata Y, Grossmann V, et al. Landscape of genetic lesions in 944 patients with myelodysplastic syndromes.
Leukemia. 2014;28(2):241-247.

4. Papaemmanuil E, Gerstung M, Bullinger L, et al. Genomic classification and prognosis in acute myeloid leukemia. N Engl J Med. 2016;374(23):2209-2221.

5. Strupp C, Nachtkamp K, Hildebrandt B, et al. New proposals of the WHO working group (2016) for the diagnosis of myelodysplastic syndromes (MDS): Characteristics of refined MDS types. Leuk Res. 2017;57:7884.

6. Obeng EA, Chappell RJ, Seiler M, et al. Physiologic expression of Sf3b1K700E causes impaired erythropoiesis, aberrant splic- ing, and sensitivity to therapeutic spliceosome modulation. Cancer Cell. 2016;30 (3):404-417.

7. Moran-Crusio K, Reavie L, Shih A, et al. Tet2 loss leads to increased hematopoietic stem cell self-renewal and myeloid transformation. Cancer Cell. 2011;20(1):11-24.

8. Hasegawa N, Oshima M, Sashida G, et al. Impact of combinatorial dysfunctions of Tet2 and Ezh2 on the epigenome in the pathogenesis of myelodysplastic syndrome. Leukemia. 2017;31(4):861-871.

9. Wang J, Li Z, He Y, et al. Loss of Asxl1 leads to myelodysplastic syndrome-like disease in 
mice Blood. 2014.123(4):541-553.

10. Kim E, Ilagan JO, Liang Y, et al. SRSF2 mutations contribute to myelodysplasia by mutant-specific effects on exon recognition. Cancer Cell. 2015;27(5):617-630.

11. Beachy SH, Aplan PD. Mouse models of myelodysplastic syndromes. Hematol Oncol Clin North Am. 2010;24(2):361-375.

12. Beurlet S, Chomienne C, Padua RA. Engineering mouse models with myelodysplastic syndrome human candidate genes; how relevant are they? Haematologica. 2012;98(1):10-22

13. Zhou T, Kinney MC, Scott LM, et al. Revisiting the case for genetically engineered mouse models in human myelodysplastic syndrome research. Blood. 2015;126(9):1057-1068

14. Malcovati L, Papaemmanuil E, Bowen DT, et al. Clinical significance of SF3B1 mutations in myelodysplastic syndromes and myelodysplastic/myeloproliferative neoplasms. Blood. 2011;118(24):6239-6246

15. Franks CR, Bishop D, Balkwill FR, et al. Growth of acute myeloid leukaemia as discrete subcutaneous tumours in immunedeprived mice. Br J Cancer. 1977;35(5):697700.

16. Lapidot T, Sirard C, Vormoor J, et al. A cell initiating human acute myeloid leukaemia after transplantation into SCID mice. Nature. 1994;367(6464):645-648.

17. Bonnet D, Dick JE. Human acute myeloid leukemia is organized as a hierarchy that originates from a primitive hematopoietic cell. Nat Med. 1997:3(7):730-737.

18. Nilsson L, Åstrand-Grundström I, Anderson $\mathrm{K}$, et al. Involvement and functional impairment of the CD34(+)CD38(-)Thy-1(+) hematopoietic stem cell pool in myelodysplastic syndromes with trisomy 8. Blood. 2002;100(1):259-267.

19. Kerbauy DM, Lesnikov V, Torok-Storb B, et al. Engraftment of distinct clonal MDSderived hematopoietic precursors in NOD/SCID-beta2-microglobulin-deficient mice after intramedullary transplantation of hematopoietic and stromal cells. Blood. 2004;104(7):2202-2203

20. Thanopoulou E, Cashman J, Kakagianne T, et al. Engraftment of NOD/SCID-2 microglobulin null mice with multilineage neoplastic cells from patients with myelodysplastic syndrome. Blood. 2004;103 (11):4285-4293.

21. Benito AI, Bryant E, Loken MR, et al. NOD/SCID mice transplanted with marrow from patients with myelodysplastic syndrome (MDS) show long-term propagation of normal but not clonal human precursors. Leuk Res. 2003:27(5):425-436.

22. Muguruma Y, Matsushita H, Yahata T, et al. Establishment of a xenograft model of human myelodysplastic syndromes. Haematologica. 2011;96(4):543-551.

23. Pang WW, Pluvinage JV, Price EA, et al. Hematopoietic stem cell and progenitor cell mechanisms in myelodysplastic syndromes. Proc Natl Acad Sci U S A. 2013;110(8):30113016.

24. Medyouf H, Mossner M, Jann J-C, et al. Myelodysplastic cells in patients reprogram mesenchymal stromal cells to establish a transplantable stem cell niche disease unit Cell Stem Cell. 2014;14(6):824-837.

25. Mian SA, Rouault-Pierre K, Smith AE, et al. SF3B1 mutant MDS-initiating cells may arise from the haematopoietic stem cell compartment. Nature Commun. 2015;6: 10004.

26. Rouault-Pierre K, Mian SA, Goulard M, et al Preclinical modeling of myelodysplastic syndromes. Leukemia. 2017;31(12):2702-2708

27. Yoshimi A, Balasis ME, Vedder A, et al. Robust patient-derived xenografts of MDS/MPN overlap syndromes capture the unique characteristics of CMML and JMML. Blood. 2017;130(4):397-407.

28. Zhang Y, He L, Selimoglu-Buet D, et al. Engraftment of chronic myelomonocytic leukemia cells in immunocompromised mice supports disease dependency on cytokines. Blood Adv. 2017;1(14):972-979.

29. Krevvata M, Shan X, Zhou C, et al Cytokines increase engraftment of human acute myeloid leukemia cells in immunocompromised mice but not engraftment of human myelodysplastic syndrome cells. Haematologica. 2018;103(6):959-971.

30. Meunier M, Dussiau C, Mauz N, et al. Molecular dissection of engraftment in a xenograft model of myelodysplastic syndromes. Oncotarget. 2018;9(19):1499315000.

31. Shastri A, Choudhary G, Teixeira M, et al Antisense STAT3 inhibitor decreases viability of myelodysplastic and leukemic stem cells. J Clin Invest. 2018:128(12):5479-5488.

32. Stevens BM, Khan N, D'Alessandro A, et al. Characterization and targeting of malignant stem cells in patients with advanced myelodysplastic syndromes. Nature Commun. 2018;9(1):3694

33. Smith MA, Choudhary GS, Pellagatti A, et al. U2AF1 mutations induce oncogenic IRAK4 isoforms and activate innate immun pathways in myeloid malignancies. Nat Cell Biol. 2019;21(5):640-650

34. Song Y, Rongvaux A, Taylor A, et al. A highly efficient and faithful MDS patient-derived xenotransplantation model for pre-clinical studies. Nature Commun. 2019;10(1):366

35. Shultz LD, Lyons BL, Burzenski LM, et al. Human lymphoid and myeloid cell development in NOD/LtSz-scid IL2R null mice engrafted with mobilized human hemopoitic stem cells. I Immunol. 2005;174(10): 6477-6489.

36. Wunderlich M, Chou F-S, Link KA, et al AML xenograft efficiency is significantly improved in NODI[sol]|SCID-IL2RG mice constitutively expressing human SCF, GM CSF and IL-3. Leukemia. 2010;24(10):17851788

37. Martin MG, Welch JS, Uy GL, et al. Limited engraftment of low-risk myelodysplastic syndrome cells in NOD/SCID gamma-C chain knockout mice. Leukemia. 2010;24(9): 1662-1664.

38. Wunderlich M, Brooks RA, Panchal R, et al. OKT3 prevents xenogeneic GVHD and allows reliable xenograft initiation from unfractionated human hematopoietic tissues. Blood. 2014:123(24):e134-e144.
39. Reinisch A Thomas D, Corces MR, et al. A humanized bone marrow ossicle xenotransplantation model enables improved engraftment of healthy and leukemic human hematopoietic cells. Nat Med. 2016;22(7): 812-821.

40. Antonelli A, Noort WA, Jaques J, et al. Establishing human leukemia xenograft mouse models by implanting human bone marrow-like scaffold-based niches. Blood. 2016;128(25):2949-2959

41. Abarrategi A, Foster K, Hamilton A, et al. Versatile humanized niche model enables study of normal and malignant human hematopoiesis. J Clin Invest. 2017;127(2): 543-548.

42. Reinisch A, Hernandez DC, Schallmoser K et al. Generation and use of a humanized bone-marrow-ossicle niche for hematopoietic xenotransplantation into mice. Nat Protoc. 2017;12(10):2169-2188.

43. Li AJ, Calvi LM. The microenvironment in myelodysplastic syndromes: niche-mediated disease initiation and progression. Exp Hematol. 2017:55:3-18.

44. Pronk E, Raaijmakers MHGP. The mesenchymal niche in MDS. Blood. 2019;133 (10):1031-1038

45. Rongvaux A, Willinger $\mathrm{T}$, Martinek J, et al, Development and function of human innate immune cells in a humanized mouse model. Nat Biotechnol. 2014;32(4):364-372

46. Herndler-Brandstetter D, Shan L, Yao Y, et al. Humanized mouse model supports development, function, and tissue residency of human natural killer cells. Proc Natl Acad Sci U S A. 2017;114(45):E9626-E9634.

47. Geyh S, Oz S, Cadeddu RP, et al. Insufficient stromal support in MDS results from molecular and functional deficits of mesenchymal stromal cells. Leukemia. 2013;27(9):18411851

48. Schroeder T, Geyh S, Germing U, et al Mesenchymal stromal cells in myeloid malig nancies. Blood Res. 2016:51(4):225-232.

49. Poon Z, Dighe N, Venkatesan SS, et al. Bone marrow MSCs in MDS: contribution towards dysfunctional hematopoiesis and potential targets for disease response to hypomethylating therapy. Leukemia. 2019;33(6):1487-1500.

50. James S, Fox J, Afsari F, et al. Multiparameter Analysis of human bone marrow stromal cells identifies distinct immunomodulatory and differentiation-competent subtypes. Stem Cell Reports. 2015;4(6):1004-1015

51. Chen Y, Jacamo R, Shi YX, et al. Human extramedullary bone marrow in mice: novel in vivo model of genetically controlled hematopoietic microenvironment. Blood. 2012;119(21):4971-4980.

52. Passaro D, Abarrategi A, Foster K, et al. Bioengineering of humanized bone marrow microenvironments in mouse and their visualization by live imaging. J Vis Exp. 2017;(126). e55914.

53. Teofili L, Martini M, Nuzzolo ER, et al. Endothelial progenitor cell dysfunction in myelodysplastic syndromes: possible contribution of a defective vascular niche to myelodysplasia. Neoplasia. 2015;17(5):401 409. 